\title{
Effect of HLA type and hypocomplementaemia on the expression of parvovirus arthritis: one year follow up of an outbreak
}

\author{
N S T Gendi, K Gibson, B P Wordsworth
}

\begin{abstract}
Objectives-To determine the effect of HLA type and hypocomplementaemia on the duration and severity of joint involvement in parvovirus infection (HPV).

Methods-Forty seven patients were selected on a geographical basis from 83 with proven HPV infection during an outbreak that occurred in Oxfordshire in 1993. They were contacted by questionnaire a year later. Thirty five patients were available for examination and blood sampling. Subjects were typed for HLA-DRB1 alleles and HLA-B27 status. Immunological profiles, including C3 and C4 complement components, were determined.

Results-Joint symptoms occurred in all patients. They resolved within a week in 12 patients and persisted beyond one year in 19. On review, none had a picture of rheumatoid arthritis, but three patients had developed carpal tunnel syndrome. Decreased C4 was found in four. The HLA frequencies were similar to those in controls; however, joint symptoms persisted for more than one week in all HLADR4 positive patients $(p=0 \cdot 009)$. There was no relation between the severity of joint symptoms and either HLA type, or hypocomplementaemia.

Conclusions-Joint symptoms are common in parvovirus infection and the presence of HLA-DR4 may be associated with persistence of joint symptoms beyond one week. This study revealed no evidence of progression to rheumatoid arthritis.
\end{abstract}

(Ann Rheum Dis 1996; 55: 63-65)

Rheumatology Department, Nuffield Orthopaedic Centre,

Oxford,

United Kingdom

NS T Gendi

K Gibson

B P Wordsworth

Correspondence to: N S T Gendi,

Rheumatology Department, Basildon Hospital,

Nether Mayne,

Basildon,

Essex SS16 5NL,

United Kingdom.

Accepted for publication

31 August 1995
The B19 strain of the human parvovirus (HPV) is a small single stranded DNA virus that has recently been linked to several clinical syndromes. It frequently causes a self limiting nondestructive arthritis in infected adults. The duration of joint symptoms varies between a few days of arthralgia to months or even years of persistent arthritis. ${ }^{1}$ Progression to erosive rheumatoid arthritis (RA) has been reported occasionally. ${ }^{23}$ Arthritis follows the stage of viraemia, suggesting an immune mediated pathogenesis. Immunological abnormalities including positive rheumatoid factor, antinuclear antibody (ANA), anti-DNA, antilymphocyte antibodies, and hypocomplementaemia have been reported. ${ }^{14-6}$ Hypocomplementaemia may be associated with immune complex formation in the acute phase of illness, or it may represent a pre-existing abnormality that predisposes to the development of arthritis. It is also conceivable that the expression of arthritis is influenced by the major HLA antigens: an increased frequency of HLA-DR4 and absence of DR1 in HPV arthritis has been reported previously, ${ }^{7}$ and an association with HLA-B27 has also been suggested. ${ }^{8}$ The aim of this study was to determine the effect of HLA antigens and hypocomplementaemia on the severity and persistence of joint involvement in HPV infection.

\section{Patients and methods}

During an outbreak of HPV in 1993, two patients presented with persistent HPV arthritis and decreased concentrations of $\mathrm{C} 3$ and C4 complement. At presentation, the first patient had a C3 concentration of $540 \mathrm{mg} / 1$ (normal value (NV) 650-1900 mg/l) and a C4 concentration <50 mg/l (NV 150-500 mg/l); DNA binding was increased, anticardiolipin antibody (IgG) was $15 \mathrm{U} / \mathrm{ml}$ (NV 0-10 U/ml), and the patient had a rheumatoid factor titre of 80 and an ANA titre of 40 . The second patient had a C 3 concentration of $590 \mathrm{mg} / \mathrm{l}, \mathrm{C} 4$ of $60 \mathrm{mg} / \mathrm{l}$ and a rheumatoid factor titre of 80 . These immunological abnormalities had all resolved by six months, except the decreased C4 concentration in the first patient, which persisted up to one year of follow up.

Examination of the records of the virology department in Oxford revealed that there were 83 positive cases (high titre of IgM antibody to B19-HPV by radioimmunoassay) during January to June 1993. Most of the patients were referred for the test by their general practitioners. One year later, 55 of these subjects were selected on a geographical basis and approached through a questionnaire (three patients who had fetal loss as a result of HPV infection were excluded). Of these, 45 patients responded. Among the total of 47 patients (including the two index cases), 43 were women. The age range was $21-55$ years (mean 37). Thirty five of the group agreed to undergo further assessment, examination and blood sampling.

HLA-DRB 1 alleles were typed using sequence specific oligonucleotide probes (SSOP) and polymerase chain reaction (PCR) amplification of genomic DNA as described 
previously. 9 PCR combined with HLA-B27 SSOP were used to define the presence or absence of this allele as also described previously. ${ }^{10} \mathrm{C} 3$ and $\mathrm{C} 4$ complement concentrations were measured by nephelometry (Array nephelometer, High Wycombe, UK). A latex slide agglutination test (Hoechest, Hounslow, UK) was used to screen for IgM rheumatoid factor and, if positive, the titre was determined by the rheumatoid arthritis precipitin assay (RAPA) gel bead agglutination assay (Fujirebio Inc, Mast Diagnostics, Bootle, UK). ANA was determined by indirect immunofluorescence on young rat liver, using IgG conjugate (Incstar, Wokingham, UK).

Patients were grouped according to the duration of symptoms. The limits of one week, two months, and until review were chosen arbitrarily to define short, medium, and prolonged duration of symptoms.

\section{STATISTICAL ANALYSIS}

Fisher's exact test was used to assess the significance of differences between groups.

\section{Results}

All 47 patients contacted had joint symptoms at onset (pain in 44 , stiffness in 44 , and swelling in 39). The joints affected included the hands ( 35 patients), knees (23), ankles (22), wrists (20), elbows (14), feet (14), shoulders (nine), and hips (eight). Symptoms lasted for one week or less in 12 patients and persisted for a minimum of one year in 19 (pain in 15, stiffness in 15, swelling in eight). Applying the 1987 ARA criteria for $\mathrm{RA}^{11}$ retrospectively, one, eight, two, one, and six patients satisfied $5,4,3,2$, and 1 of the criteria, respectively. Twelve patients did not wish to attend for inteview, but details of their symptoms as given in response to the quesionnaire appeared similar to those of the group overall. None of those examined had definite synovitis or deforming arthritis. Three patients developed carpal tunnel syndrome that required decompression in two. Five had low back pain as part of their illness, and an additional five had apparently unrelated back pain. Transient rashes occurred in 34 patients,

$H L A-D R$ frequencies in relation to duration of joint symptoms

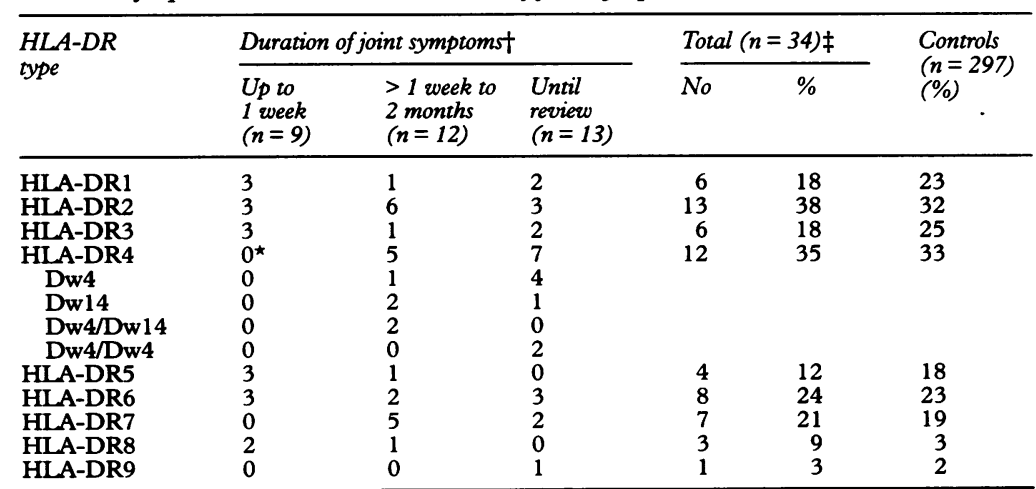

fAll patients whose symptoms persisted beyond two months had symptoms at one year. $\$$ The blood specimen of the 35 th subject was lost.

${ }^{\star}$ Significant difference $(p=0.009)$ compared with patients whose symptoms persisted beyond one week. and seven described generalised swelling of the extremities, with temporary weight gain documented by one patient.

The HLA-DR frequencies in patients were similar to those in controls (table). There was a statistically significant relation between HLA-DR4 and symptom persistence beyond one week $(p=0.009)$, but not beyond two months $(p=0.06)$. There was no relation between the HLA type and the severity of symptoms as determined by the number of ARA criteria satisfied, or by a general estimate by the researcher. HLA-B27 was positive in two patients; one of them had back pain that predated her HPV infection.

At one year, $\mathrm{C} 4$ concentration was decreased in four patients (HLA types: DR3/DR3, DR3/DR5 and DR4/DR2 in two patients), but C3 was normal in all. There was no correlation between hypocomplementaemia and persistence or severity of joint symptoms. Rheumatoid factor was positive with a titre of 160 in one patient and 80 in another. Both these patients had joint symptoms persisting until review and, respectively, would have satisfied 5 and 4 of the 1987 ARA criteria applied retrospectively; however, they did not fulfill the criteria for RA when examined at one year. An ANA titre of 40 was present in two patients.

\section{Discussion}

There has been an interest in HPV as a possible infectious trigger for RA. Joint symptoms in the form of pain, stiffness, and swelling can persist, and the ARA criteria for RA may be transiently satisfied. However, follow up studies, including ours, have generally failed to demonstrate progression to an erosive deforming arthritis. ${ }^{1}$ Such progression, when it has occasionally been described, ${ }^{2}{ }^{3}$ was probably coincidental. It is possible that HPV infection may lead to RA many years after the original infection. However, the results of studies attempting to link RA to previous HPV exposure are conflicting. ${ }^{2} 1213$

Three of our patients developed carpal tunnel syndrome. This is consistent with the reported association of HPV with finger paraesthesia and slowing of nerve conduction velocities. ${ }^{14}$ HPV may therefore precipitate carpal tunnel syndrome, presumably as a result of synovitis and local swelling.

Even in diseases with a known single aetiology, manifestations vary between individuals. This variability of disease expression is likely to be related to the genetic make up of each individual. In the case of HPV arthritis, it is logical to look at genes already known to be associated with RA. Klouda $e t a l^{7}$ found an increased frequency of HLA-DR4 in 18 patients with HPV arthritis (67\% compared with $36 \%$ in controls). A subsequent review of 27 patients by the same group ${ }^{15}$ revealed a nonsignificant increased frequency of HLA-DR4 $(52 \%)$. In contrast, we did not observe an excess HLA-DR4 in our patient group as a whole. This could be explained by differences between the studies regarding patient 
recruitment, as ours were referred by general practitioners for serological testing, in contrast with direct referral to an early synovitis clinic. $^{715}$ Although we selected patients from the cohort on a geographical basis, some bias towards severity could have occurred at the levels of general practitioner referrals and patient responses to the questionnaire. However, our study does suggest a weak effect of HLA-DR4 on the persistence of joint symptoms. The frequency of HLA-DR4 was $48 \%$ in those whose symptoms persisted beyond one week, but was completely absent in those whose symptoms resolved within one week. Although there is documented association of severity of RA with certain genotypes (for example Dw4/Dw14), the numbers in our study were too small to find a similar relation in HPV arthritis. We could not confirm the reported absence of HLA-DR $1,{ }^{75}$ or the suggested relation with HLA-B27. ${ }^{8}$

Immunological abnormalities are known to occur in HPV infection, ${ }^{14-6}$ but we found that such abnormalities persisted only in a minority at one year. The persistence of decreased concentrations of $\mathrm{C} 4$ in four of our patients suggests a pre-existing abnormality that was accentuated during the acute illness in one of our patients; however, the number of patients with decreased $\mathrm{C} 4$ was too small to permit us to draw any conclusions. To define the exact relation of hypocomplementaemia to the pathogenesis of HPV arthritis, it may be helpful to study the profile of complement levels throughout the illness, combined with typing for complement null alleles.
We are grateful to Dr J B Kurtz for allowing us access to the records of the virology department in Oxford.

1 White D G, Woolf A D, Mortimer P P, Cohen B Blake D R, Bacon P A. Human parvovirus arthropathy. Lancet 1985; i: 419-21.

2 Cohen B J, Buckley M M, Clewley J P, Jones V E Puttick A H, Jacoby $R$ K. Human parvovirus infection in early rheumatoid and inflammatory arthritis. Ann Rheum Dis 1986; 45: 832-8.

3 Tyndall A, Jelk W, Hirsch H H. Parvovirus B19 and erosive polyarthritis [letter]. Lancet 1994; 343: 480-1.

4 Kalish R A, Knopf A N, Gary G W, Canoso J J. Lupuslike presentation of human parvovirus B19 infection. f Rheumatol 1992; 19: 169-71.

5 Naides S J, Field E H. Transient rheumatoid factor positivity in acute human parvovirus B19 infection. Arch Intern Med 1988; 148: 2587-9.

6 Solninka C A, Anderson M J, Laskin C A. Anti-DNA and antilymphocyte antibodies during acute infection with human parvovirus B19. ₹ Rheumatol 1989; 16: 777-81.

7 Klouda P T, Corbin S A, Bradley B A, Cohen B J, Woolf A D. HLA and acute arthritis following human parvovirus infection. Tissue Antigens 1986; 28: 318-9.

8 Jawad A S. Persistent arthritis after human parvovirus B19 infection [letter]. Lancet 1993; 341: 494

9 Wordsworth B P, Allsop C E, Young R P, Bell J I. HLA-DR typing using DNA amplification by the polymerase chain reaction and sequential hybridization to sequence-specific oligonucleotide probes. Immunogenetics 1990; 32: 413-8.

10 Hill A V, Allsop C E, Kwiatkowski D, Anstey N M, Greenwood B M, McMichael A J. HLA class I typing by PCR: HLA-B27 and African B27 subtype. Lancet 1991; 337: $640-2$.

11 Armett F C, Edworthy S M, Bloch D A, et al. The American Rheumatism association 1987 revised criteria for the Rheumatism association 1987 revised criteria for the 1988; 31: 315-24.

12 Hajeer A H, MacGregor A J, Rigby A S, Ollier W E R, Carthy D, Silman A J. Influence of previous exposure to human parvovirus B19 infection in explaining susceptibility to rheumatoid arthritis: an analysis of disease discordant twin pairs. Ann Rheum Dis 1994; 53: 137-9.

13 Nikkari S, Luukkainen R, Mottonen T, et al. Does parvoviros B19 have a role in rheumatoid arthritis? Ann Rheum Dis 1994; 53: 106-11.

14 Faden $\mathrm{H}$, Gary G W Jr, Korman M. Numbness and tingling of fingers associated with parvovirus B19 infection [letter]. F Infect Dis 1990; 161: 354-5.

15 Woolf A D, Campion G V, Klouda P, Chiswick A Cohen B, Dieppe P A. HLA and the manifestations of human parvovirus B19 infection [abstract]. Arthritis Rheum 1987; 30: S52. 